\title{
The NA62 Calorimeter Level 0 Trigger Operation and Performances
}

\author{
R. Aliberti (a), R. Ammendola (b), D. Battista (b), M. Barbanera ${ }^{(c)}$, M. Bizzarri $^{(d)}$, \\ V. Bonaiuto (b), A. Ceccucci (e), B. Checcucci (d), N. De Simone (e), R. Fantechi (c),

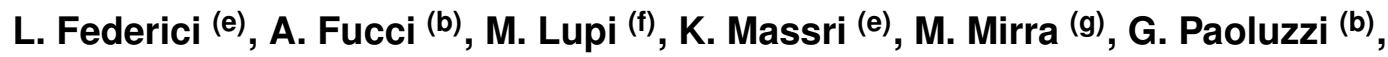 \\ A. Papi (d), C. Parkinson (h), E. Pedreschi (c), R. Piandani (c), M. Piccini (d), \\ G. Ruggiero $^{(i)}$, V. Ryjov ${ }^{(e)}$, A. Salamon ${ }^{*((b)}$, G. Salina ${ }^{(b)}$, F. Sargeni ${ }^{(b)}$, A. Sergi ${ }^{(h)}$, \\ D. Soldi ${ }^{(j)}$, F. Spinella ${ }^{(c)}$, S. Venditti ${ }^{(k)}$, M. Zamkovsky ${ }^{(l)}$ \\ (a) Johannes Gutenberg Universitaet Mainz, Mainz, DE \\ (b) INFN and Università degli Studi di Roma Tor Vergata, Roma, IT \\ (c) INFN and Università di Pisa, Pisa, IT \\ (d) INFN and Università degli Studi di Perugia, Perugia, II \\ (e) CERN, European Organization for Nuclear Research, Geneva, CH \\ (f) Johann Wolfgang Goethe University, Frankfurt, DE \\ (g) INFN and Università degli Studi di Napoli Federico II, Napoli, IT \\ (h) University of Birmingham, Birmingham, UK \\ (i) Lancaster University, Lancaster, UK \\ (j) INFN Sezione di Torino, Torino, IT \\ (k) CAEN, Viareggio, IT \\ (l) Charles University, Prague, $C Z$
}

\begin{abstract}
The NA62 experiment at the CERN SPS aims to measure the branching ratio of the very rare kaon decay $K^{+} \rightarrow \pi^{+} v \bar{v}$, collecting $\sim 100$ events with a $10 \%$ background to make a stringent test of the Standard Model. The calorimeter level 0 trigger is used to suppress one of the main backgrounds, the $K^{+} \rightarrow \pi^{+} \pi^{0}$ decay, and to select events with a $\pi^{+}$in the final state. The calorimeter level 0 trigger identifies clusters in the electromagnetic and hadronic calorimeters. It prepares timeordered lists of reconstructed clusters together with the arrival time, position, and energy measurements of each cluster. It also provides trigger decisions based on complex combinations of energy and cluster multiplicity. The main parameters of the trigger processor are the high design hit rate $(30 \mathrm{MHz})$ and the required single cluster time resolution $(1.5 \mathrm{~ns})$. The calorimeter trigger processor is a parallel system composed of 37 boards, 111 mezzanines and 221 high-performance programmable devices housed in three $9 \mathrm{U}$ crates. The design, operation and performances of the calorimeter level 0 trigger are presented.
\end{abstract}

The European Physical Society Conference on High Energy Physics

5-12 July

Venice, Italy

\footnotetext{
${ }^{*}$ Speaker.

${ }^{\dagger}$ E-mail: andrea.salamon@ roma2.infn.it
} 


\section{The NA62 experiment at the CERN SPS}

The NA62 experiment [1] aims to measure the very rare kaon decay $K^{+} \rightarrow \pi^{+} v \bar{v}$, collecting $O(100)$ events with a $10 \%$ background to make a stringent test of the Standard Model and deepen the knowledge of the CKM matrix.

The $K^{+} \rightarrow \pi^{+} v \bar{v}$ decay is highly suppressed, well know from the theoretical point and very sensitive to many New Physics models. The Standard Model branching ratio prediction for $\mathrm{K}^{+} \rightarrow$ $\pi^{+} v \bar{v}$ is $(8.4 \pm 1.0) \times 10^{-11}[2,3]$.

The NA62 detector [4], currently taking data at the CERN SPS North Area, is composed of (see Figure 1): a differential Cherenkov counter (KTAG), a beam tracker (GTK), a charged particle detector (CHANTI), magnetic spectrometer based on straw drift tubes (STRAW), a Cherenkov detector (RICH), a charged particle hodoscope (CHOD), a photon veto system composed of different detectors covering the various angular regions (LAV, LKR, IRC, SAC) and a muon-veto system based on two hadronic calorimeters (MUV1, MUV2) and a plane of scintillating tiles (MUV3).

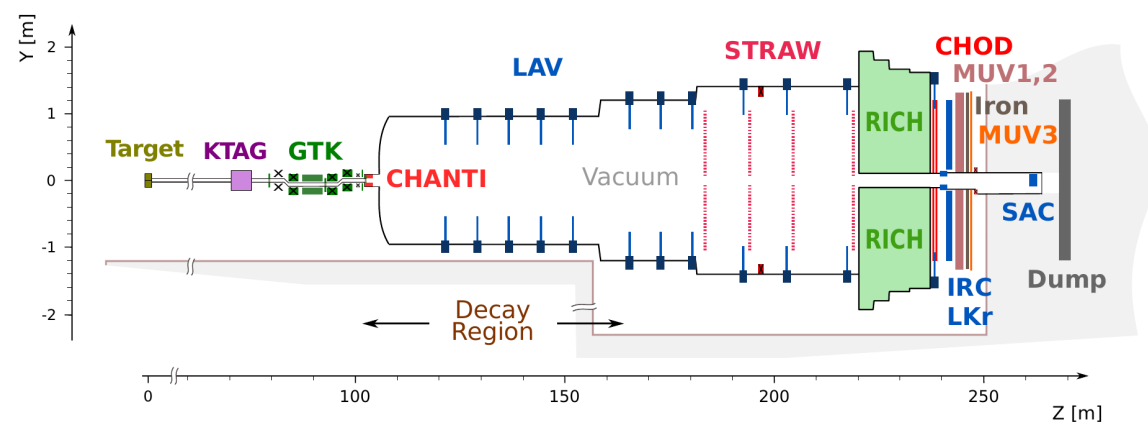

Figure 1: Schematic drawing of the NA62 detector at the CERN SPS.

\section{Trigger and Data Acquisition system}

In order to extract the small number of interesting decays from a very intense particles flux a complex high-performance three level trigger and data acquisition system was designed [5]. The level 0 trigger system is based on a small number of sub-detectors (CHOD, RICH, LAV, MUV3 and all the calorimeters) and is performed by dedicated custom hardware modules, while level 1 and level 2 software triggers are executed on a dedicated PC farm.

\section{Electromagnetic and hadronic calorimeters}

An efficient photon veto system was designed to suppress the background from $K^{+} \rightarrow \pi^{+} \pi^{0}$ decay.

In the 1-8.5 mrad angular region the NA48 electromagnetic liquid krypton calorimeter ( $\mathrm{LKr}$ ) is used [6], which is a quasi-homogeneous ionization device characterized by excellent time and energy resolution. 


\begin{tabular}{lcc}
\hline \hline Detector & Physical channels & Trigger channels \\
\hline LKr & 13,248 & 864 \\
IRC & 4 & 1 \\
SAC & 4 & 1 \\
MUV1 & 176 & 6 (vertical) +6 (horizontal) \\
MUV2 & 88 & 3 (vertical) +3 (horizontal) \\
\hline Total & 13,520 & 884 \\
\hline \hline
\end{tabular}

Table 1: The calorimeter $\mathrm{L} 0$ trigger receives digitized data from five NA62 calorimeters: $\mathrm{LKr}$, IRC and SAC electromagnetic calorimeter and MUV1 and MUV2 hadronic calorimeters.

Two detectors are used to cover the angular region below $1 \mathrm{mrad}$ : the intermediate-ring calorimeter (IRC) and the small-angle calorimeter (SAC) which are shashlyk calorimeters, with lead and plastic-scintillator plates traversed by wavelength-shifting fibres.

The hadronic calorimeter is a sampling calorimeter made from alternate layers of iron and scintillator divided into two independent detectors: the front detector (MUV1), newly built for the NA62 experiment, and the back detector (MUV2) inherited from the NA48 experiment.

All these detectors are read-out by the so-called Calorimeter REAd-out Modules [7] (CREAMs) which provides $40 \mathrm{MHz} 14$ bit sampling, data buffering, optional zero suppression and programmable trigger sums for the level 0 electromagnetic calorimeter trigger processor.

The angular decay region from $50 \mathrm{mrad}$ down to $8.5 \mathrm{mrad}$ is covered by the Large Angle Veto (LAV) which is read-out by its own dedicated digitization electronics.

\section{Calorimetric trigger implementation}

The calorimeter level 0 trigger identifies clusters from the electromagnetic ( $\mathrm{LKr}$, IRC and SAC) and hadronic (MUV1 and MUV2) calorimeters (see Table 4), all read-out through the CREAM system. It prepares time-ordered lists of reconstructed clusters together with the arrival times, positions, and energy measurements of each cluster. Electromagnetic and hadronic cluster search is executed in parallel for each of the five detectors to provide trigger decisions based on complex energy and cluster multiplicity combinations [8,9].

The trigger processor (Figure 2) is a parallel system, composed of Front-End, Merger and Concentrator boards, all based on the TEL62 cards [10] complemented with custom mezzanine boards $[11,12]$.

The basic functions of the three stages are:

- the Front-End boards receive trigger sums from the CREAM and perform peak searches in space and compute time, position and energy for each detected peak;

- the Merger boards (only used for the LKr) receive trigger data from the Front-End boards and merge peaks from different Front-End boards into single clusters; 


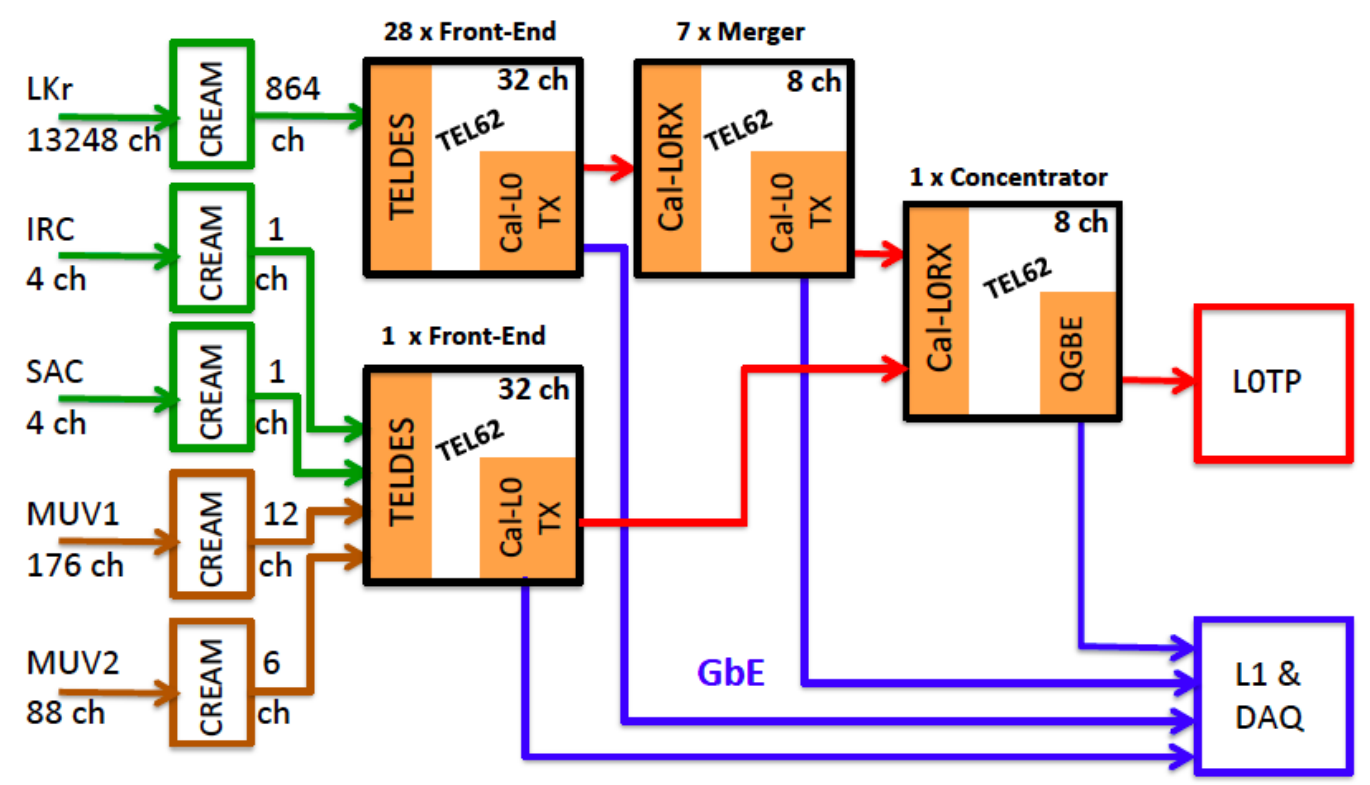

Figure 2: Schematic diagram of the calorimetric trigger processor. The system, installed in three $9 \mathrm{U}$ racks in the room above the LKr calorimeter, is composed of 37 TEL62 boards with dedicated daughter boards. Three different layers of boards are used: Front-End, Merger (only for LKr calorimeter) and Concentrator.

- the Concentrator boards receive reconstructed clusters from the five NA62 calorimeters, perform cluster counting, calculate sums for electromagnetic and hadronic energy and generates trigger primitives for the L0 Trigger Processor.

The cluster search in the $\mathrm{LKr}$ is executed in two steps with two unidimensional algorithms. The calorimeter is divided in slices parallel to the vertical axis. In the first step peaks in space and time are sought independently in each slice with a unidimensional algorithm, along such axis. In the second step different peaks which are close in time and space are merged and assigned to the same electromagnetic cluster.

The cluster search in IRC, SAC, MUV1 and MUV2 calorimeter is executed in a single step with a one-dimensional algorithm.

Reconstructed clusters from all these electromagnetic and hadronic calorimeters are put together in the last stage of the trigger processor (Concentrator board) which performs trigger algorithms based on complex energy and cluster multiplicity combinations [13,14] and sends trigger decision the the NA62 L0 Trigger Processor [15].

As such, the system also provides a coarse-grained read-out of the calorimeters that can be used in L1/L2 software trigger levels.

All together, the system is composed of 37 TEL62 boards, 111 mezzanine cards and 221 highperformance FPGAs.

\section{Calorimetric trigger operation and performances}

Given the calorimeter trigger installation environment (ECN3 experimental hall in the SPS 
North Area) and operating conditions (characterized by high hit rates), all the system parameters must be continuously monitored to guarantee expected performances.

All the processor's relevant parameters are continuously monitored by the on-board Credit Card PCs with a major emphasis on reconstructed peaks and cluster rates, FIFO over and underflow and any other anomalous condition.

One crucial point in the effective operation of the trigger is high bandwidth data transfer between different FPGAs in the same board and between different boards in the processor. In order to guarantee an error free data transmission an error monitoring and correction code was implemented for all transmissions between and inside all boards.

Radiation effects on sensitive electronic devices must be continuously monitored, too. In particular two kinds of Single Event Upsets (SEUs) are taken into account: SEUs in the FPGAs [16] and SEUs in all other not reprogrammable devices (e.g. serializer/deserializer circuits, Credit Cards PCs). Both kinds of Single Event Upsets are managed with periodic reconfiguration (for FPGAs) or power cycles (for all other not reprogrammable devices).
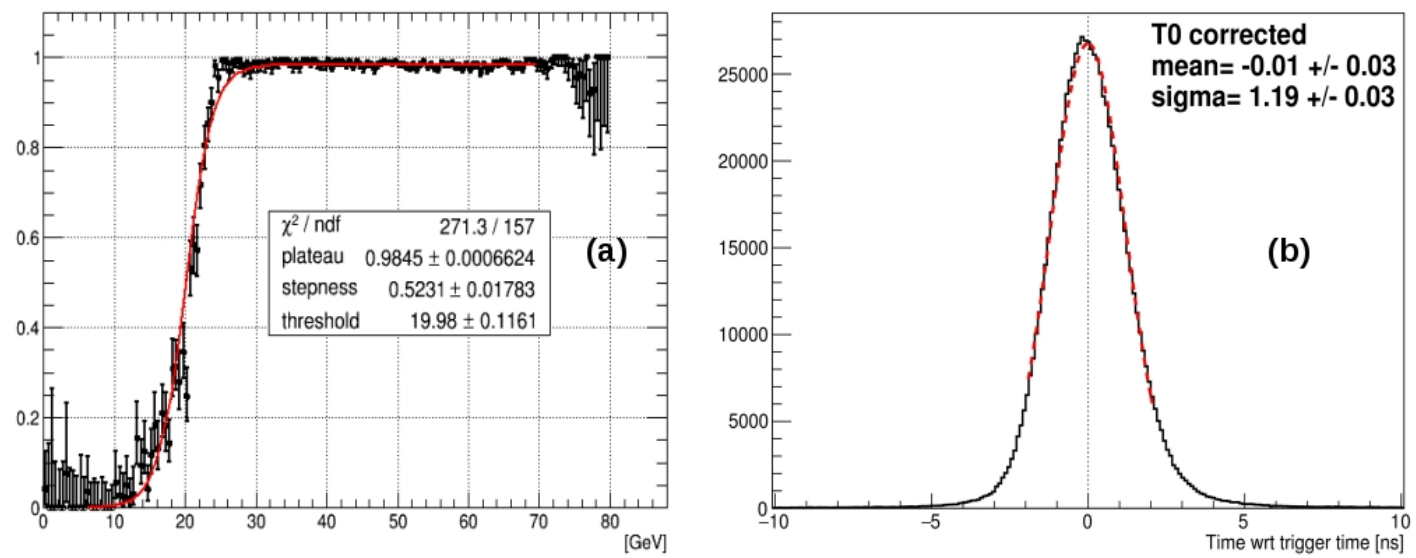

Figure 3: The calorimetric trigger $\pi^{0}$ rejection efficiency with $20 \mathrm{GeV}$ threshold is better than $98 \%$ (a) and the time resolution is better than $1.2 \mathrm{~ns}(\mathrm{~b})$.

\section{Calorimetric trigger performances}

The calorimeter L0 trigger processor is currently taking data with the NA62 experiment. It is used to veto with high efficiency and time resolution (see Figure 3 ) events with substantial electromagnetic energy in the calorimeter $\left(K^{+} \rightarrow \pi^{+} \pi^{0}\right.$ decay) and to select events for many other studies (among the others, search for lepton-flavour and lepton-number violating decays and searches for exotic particles).

The trigger processor functioned as expected with up to $100 \%$ beam intensity, no data transmission errors, no internal buffers overflows or other hardware blocking conditions were found during the high intensity runs.

\section{Conclusions}

The NA62 experiment at the CERN SPS aims to measure the branching ratio of the very rare 
kaon decay $K^{+} \rightarrow \pi^{+} v \bar{v}$ collecting $\sim 100$ events with a $10 \%$ background. The calorimeter level 0 trigger is used to suppress one of the main backgrounds, the $K^{+} \rightarrow \pi^{+} \pi^{0}$ decay and to select events for many other studies. The trigger is performing as expected. The design, operation and performances of the calorimeter level 0 trigger were presented.

\section{References}

[1] NA62 Collaboration, Proposal to Measure $K^{+} \rightarrow \pi^{+} v \bar{v}$ rare decay at the CERN SPS, CERN-SPSC-2005-013, 2005.

[2] J. Brod et al., Two-loop electroweak corrections for the decays $K \rightarrow \pi v \bar{v}$, Phys. Rev. D 83, 034030 (2011), DOI: 10.1103/PhysRevD.83.034030.

[3] A.J. Buras et al., $K^{+} \rightarrow \pi^{+} v \bar{v}$ and $K_{L}^{0} \rightarrow \pi^{0} v \bar{v}$ in the Standard Model: Status and Perspectives, JHEP 1511 (2015) 33, DOI: 10.1007/JHEP11(2015)033.

[4] NA62 Collaboration, The Beam and detector of the NA62 experiment at CERN, JINST 12 P05025, 2017, DOI: 10.1088/1748-0221/12/05/P05025.

[5] M. Sozzi, A concept for the NA62 Trigger and Data Acquisition, NA62-07-03, 2007.

[6] NA48 Collaboration, The Beam and Detector for the NA48 neutral kaon CP violation experiment at CERN, Nucl. Instrum. Methods A, A 574, 433-471, 2007, DOI: 10.1016/j.nima.2007.01.178.

[7] A. Ceccucci et al., The NA62 Liquid Krypton calorimeter readout module, JINST 6 C12017, 2011, DOI: $10.1088 / 1748-0221 / 6 / 12 / C 12017$.

[8] A. Fucci et al., The NA62 Liquid Krypton Electromagnetic Calorimeter Level-0 Trigger, Proceedings of the 13th ICATPP Conference, 2011, DOI: 10.1142/9789814405072_0077.

[9] V. Bonaiuto et al., Status of the NA62 liquid krypton electromagnetic calorimeter Level-0 trigger processor, JINST 8 C02054, 2013, DOI: 10.1088/1748-0221/8/02/C02054.

[10] B. Angelucci et al., TEL62: an integrated trigger and data acquisition board, JINST 7 C02046, 2012, DOI: $10.1088 / 1748-0221 / 7 / 02 / C 02046$.

[11] B. Checcucci et al., Development and Test Results of a Digital Data Transmission System for Liquid Krypton Calorimeter Level 0 Trigger System for the NA62 Experiment at CERN, Conference Record of 2015 IEEE Nuclear Science Symposium

[12] H. Muller et al., Quad Gigabit Ethernet plug-in card, LHCb Technical Note, (2005) https://edms5.cern.ch/document/520885

[13] D. Badoni et al., Use of FPGA embedded processors for fast cluster reconstruction in the NA62 liquid krypton electromagnetic calorimeter, JINST 9 C01010, 2014, DOI: 10.1088/1748-0221/9/01/C01010.

[14] R. Ammendola et al., The Level-0 Calorimetric Trigger of the NA62 Experiment, JINST 11 C02084, 2016, DOI: 10.1088/1748-0221/11/02/C02084.

[15] S. Chiozzi et al., Level Zero Trigger processor for the ultra rare kaon decay experiment-NA62, JINST 11 C02037, 2016, DOI: 10.1088/1748-0221/11/02/C02037.

[16] Altera Corporation, AN539: Test Methodology of Error Detection and Recovery using CRC in Altera FPGA Devices, 2015. 